\title{
Effect of Haloperidol and Risperidone on Serum Melatonin and GAP-43 in Patients with Schizophrenia: A Prospective Cohort Study
}

\author{
Rituparna Maiti ${ }^{1}$, Biswa Ranjan Mishra ${ }^{2}$, Monalisa Jena ${ }^{1}$, Archana Mishra ${ }^{3}$, Santanu Nath ${ }^{2}$ \\ Departments of ${ }^{1}$ Pharmacology and ${ }^{2}$ Psychiatry, All India Institute of Medical Sciences (AllMS), Odisha, ${ }^{3}$ Department of Pharmacology, All India \\ Institute of Medical Sciences (AIIMS), New Delhi, India
}

\begin{abstract}
Objective: Serum melatonin, a biomarker of circadian rhythm, can upregulate Growth-associated protein 43 (GAP-43) which is involved in neural regeneration and plasticity. The present study was conducted to investigate the adequacy of the first-line antipsychotic drugs to improve sleep and circadian rhythm disruptions by assessing the effect of haloperidol and risperidone on serum melatonin and GAP-43 in schizophrenia.

Methods: In this cohort study, 100 schizophrenic patients were recruited, and clinical evaluations were done using the Positive and Negative Syndrome Scale (PANSS) and the Pittsburgh sleep quality index (PSQI). The patients with predominantly positive symptoms taking haloperidol (Group I) and patients with predominantly negative symptoms taking risperidone (Group II) were admitted and serum melatonin, arylalkylamine N-acetyltransferase, GAP-43 and urinary melatonin were estimated. After 8 weeks, all clinical and biochemical parameters were repeated.

Results: Serum melatonin (2:00 hours) was significantly decreased in both haloperidol $(2.42 ; 95 \%$ confidence interval [95\% Cl]: $0.67-4.17 ; p=0.008)$ and risperidone group $(3.40 ; 95 \% \mathrm{Cl}: 0.54-6.25 ; p=0.021)$. Urinary melatonin was significantly decreased in both haloperidol $(p=0.005)$ and risperidone group $(p=0.014)$. PSQI score was significantly increased in both haloperidol $(p=0.001)$ and risperidone group $(p=0.003)$. Serum GAP-43 was significantly decreased in both haloperidol and risperidone group $(p<0.001)$. PANSS decreased significantly in both the groups and there was a significant negative correlation between serum melatonin at 2:00 hours and PANSS $(r=-0.5)$ at baseline.

Conclusion: Monotherapy with haloperidol and risperidone can achieve symptomatic improvement but cannot improve sleep and circadian rhythm disturbances in schizophrenia.
\end{abstract}

KEY WORDS: Schizophrenia; Melatonin; GAP-43; Haloperidol; Risperidone.

\section{INTRODUCTION}

Schizophrenia is a complex disorder involving multiple neurochemical systems. Multiple theories have been suggested over the years that aim to gestate the pathophysiological processes of schizophrenia, including sleep and circadian rhythm dysregulation [1-6]. Sleep disturbance and abnormal sleep-wake cycles are common in schizophrenic and can be sufficiently severe to warrant medical attention [7-11]. The sleep disturbances in schizophrenia

Received: June 19, 2020 / Revised: August 6, 2020

Accepted: September 7, 2020

Address for correspondence: Biswa Ranjan Mishra

Department of Psychiatry, All India Institute of Medical Sciences (AIIMS), Sijua, PO Dumuduma, Bhubaneswar 751019, Odisha, India E-mail: brm1678@gmail.com

ORCID: https://orcid.org/0000-0003-0537-9454 appear to be caused by abnormal circadian and abnormal pineal melatonin functions implicated in the pathophysiology of schizophrenia [12-15]. Previous studies have reported decreased melatonin level in schizophrenic patients [16-19]. Pronounced sclerosis and gliosis of pineal glands or diminished activity of enzymes in the melatonin biosynthesis pathway may decrease melatonin synthesis and/or produce abnormal melatonin compounds [20,21].

Growth-associated protein 43 (GAP-43) expression is vital for the integrity of neural circuitry, a process thought to be perturbed in schizophrenia $[22,23]$. Changes in GAP-43 protein levels and GAP-43 mRNA levels have been demonstrated in different parts of the brain of schizophrenic patients but some conflicts exist in the previous results [24-30]. The previous study by Juan et al. [31] also found that melatonin upregulates GAP-43 which ac-

@) This is an Open-Access article distributed under the terms of the Creative Commons Attribution Non-Commercial License (http://creativecommons.org/licenses/by-nc/4.0) which permits unrestricted non-commercial use, distribution, and reproduction in any medium, provided the original work is properly cited. 
counts for improving neuroplasticity, reorganization of synaptic connections, a process thought to be perturbed in schizophrenia [32].

Although melatonin level and GAP-43 has been studied and implicated in the pathophysiology of schizophrenia, till date, except chlorpromazine and olanzapine, the effect of other antipsychotics on serum melatonin and GAP-43 in schizophrenia has not been evaluated $[18,33]$. So, the present study has been conducted with an objective to evaluate the effect of a typical (haloperidol) and an atypical (risperidone) antipsychotics on serum melatonin and GAP-43 in schizophrenia.

\section{METHODS}

The present study was conducted following Indian Council of Medical Research's National ethical guidelines for biomedical and health research involving human participants (2017) after getting the approval of the institutional ethics committee (IM-F/Pharm/15/04). Written informed consent was taken from the legally authorized representatives of the recruited patients.

\section{Study Population and Eligibility}

Patients aged 18-50 years, of either sex attending Psychiatry outpatient department of AIIMS, Bhubaneswar with schizophrenia were screened for the study and enrolment was done according to inclusion and exclusion criteria. Patients with the clinical diagnosis of Schizophrenia (Diagnostic and Statistical Manual of Mental Disorders 5th edition) who received either haloperidol or risperidone according to predominant scoring in Positive and Negative Syndrome Scale (PANSS) were recruited in the study. The recruited patients were treatment naïve or did not receive any treatment for at least 4 weeks before recruitment. Patients with hebephrenic and catatonic schizophrenia, patients diagnosed with a schizoaffective disorder or schizophrenia with florid affective symptoms, patients who were highly agitated, suicidal, homicidal who needs immediate treatment were excluded. Patients who are already under treatment for the presenting conditions or have already received benzodiazepines/z compounds or with comorbid substance abuse or history of organicity and pregnant and nursing women were also excluded.

\section{Study Design}

The present study was a prospective cohort study and was conducted in a single centre. After screening 100 consecutive patients were enrolled following inclusion and exclusion criteria. After recruitment, a detailed history was taken, clinical evaluations were done, and the quality of sleep was assessed using Pittsburg sleep quality index (PSQI). Depending on the predominant PANSS score, the first group of patients with predominantly positive symptoms were on haloperidol (4 mg) and the second group of patients with predominantly negative symptoms were on risperidone $(2 \mathrm{mg})$. Throughout the study period of 8 weeks, the patients were on monotherapy and no adjunctive psychotropic drugs were prescribed. All recruited patients were admitted, and the blood sample was collected at 14:00 hours and 02:00 hours for the estimation of serum melatonin, serum GAP-43 and serum arylalkylamine $\mathrm{N}$-acetyltransferase (AANAT). Urinary melatonin was assessed with the first-morning sample of urine. After 4 weeks, patients were followed up for clinical evaluation and adverse drug reactions (if any). After 8 weeks, patients were again admitted for one day for estimation of serum melatonin, urinary melatonin and serum GAP-43. At 8 weeks follow-up, clinical evaluation was repeated with PANSS scoring and PSQI scoring. Serum melatonin and serum GAP-43 was estimated by ELISA using commercially available Human ELISA kit and urinary melatonin by high performance liquid chromatography.

\section{Outcome Measures}

\section{PANSS}

The patient is rated from 1 to 7 on 30 different symptoms based on the interview. Each of the 30 items has a specific definition and detailed anchoring criteria for rating. Out of 30 items, 7 items stand for a positive scale, 7 a negative scale, and the remaining 16 a general psychopathology scale. The scores are calculated by summation of ratings across the items. Therefore, the scoring ranges from 7 to 49 for the positive and negative Scales, and from 16 to 112 for the general psychopathology scale. PANSS scoring was done by a single investigator to avoid inter-rater variability [34].

\section{Pittsburg sleep quality index}

The PSQI is a self-rated questionnaire which assesses 
the quality of sleep and sleep-disturbances over a 1-month time interval. Nineteen individual items yield seven domain scores: subjective sleep quality, sleep latency, sleep duration, habitual sleep efficiency, sleep disturbances, use of sleeping medication, and daytime dysfunction. The sum of scores for these seven domains yields one global score. The questionnaire contains 19 questions, each weighted on a $0-3$ interval scale and a lower score reflects better sleep quality [35].

\section{Serum melatonin, AANAT and GAP-43}

Serum melatonin, AANAT and GAP-43 were measured by using human ELISA kit using double-antibody sandwich enzyme-linked immunosorbent assay technique.

\section{Urinary melatonin}

For estimation of urinary melatonin metabolite (6-sulphatoxymelatonin or 6aMTs), morning first void sample was collected, and $8 \mathrm{ml}$ urine was saturated using $1 \mathrm{~g}$ boric acid and preserved at $-80^{\circ} \mathrm{C}$ until estimation [36]. Melatonin standards were obtained from Sigma-Aldrich and estimation was done by HPLC.

\section{Safety Evaluation}

The occurrence of adverse events (AEs) was assessed by the nondirective questioning of the patient at the follow-up visit. Patients had free access to the investigators for reporting any adverse effects experienced by them. All AEs were recorded and opinion about the causal relationship to haloperidol and risperidone.

\section{Statistical Analysis}

Continuous variables have been represented as a mean \pm standard deviation/standard error of the mean and categorical variables as a percentage. Comparison of means between the groups was performed using unpaired $t$ test/ Wilcoxon rank-sum test and within the group by two-sided paired $t$ test/Wilcoxon signed-rank test. Fisher's exact test was used for comparing categorical variables between the groups. Intention to treat (ITT) was conducted by replacing missing values using multiple imputations and the pooled data was used for analysis. Pearson product-moment correlation coefficient was calculated for measuring the correlation between outcome measures. Possible variables of melatonin level like smoking, season (summer/winter), age group were assessed by linear re-

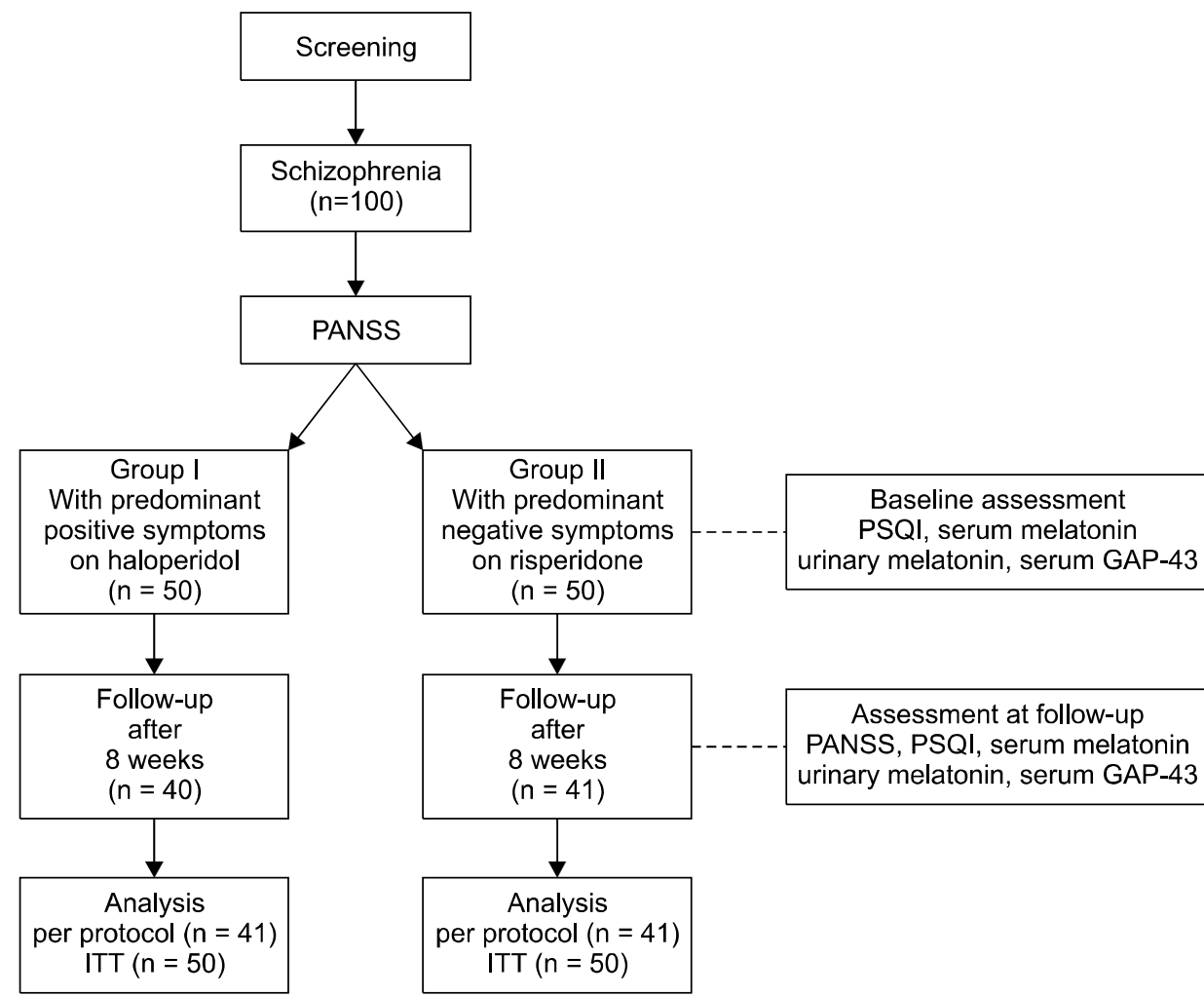

Fig. 1. Study flow chart. PANSS, Positive and Negative Syndrome Scale; ITT, intention to treat; PSQI, Pittsburgh sleep quality index; GAP-43, growth-associated protein 43. 
gression analysis. Statistical analyses will be performed using statistical software SPSS 20.0 (IBM Corp., Armonk, NY, USA) considering a significance level of $p<0.05$.

\section{Sample size calculation}

A sample size of 42 in each group was powered at $80 \%$ to detect a difference of $3 \mathrm{pg} / \mathrm{ml}$ in the change in serum melatonin. The alpha error allowed was 0.05 and the standard deviation was assumed to be 4.8 in each group based on previous studies.

\section{RESULTS}

\section{Patient Demographics and Baseline Characteristics}

The recruitment process was started in October 2016 and the study was completed by March 2018. Out of 100 schizophrenia patients recruited, 81 patients completed both the follow-ups. Ten patients in the haloperidol group and nine patients in the risperidone group were lost to follow-up at the end of 8 weeks (Fig. 1). Six out of 10 patients in the haloperidol group developed extrapyramidal symptoms and excluded from the study. The reason for the loss of follow-up of the other 13 patients was not known. There was no significant difference between the groups at

Table 1. Baseline demographic data and clinical characteristics

\begin{tabular}{lccc}
\hline \multicolumn{1}{c}{ Characteristic } & $\begin{array}{c}\text { Haloperidol } \\
\text { group }\end{array}$ & $\begin{array}{c}\text { Risperidone } \\
\text { group }\end{array}$ & $p$ value \\
\hline $\begin{array}{l}\text { Number of patients } \\
\text { recruited }\end{array}$ & 50 & 50 & \\
Male:Female ratio & $33: 17$ & $24: 26$ & 0.106 \\
Mean age (yr) & $33.44 \pm 10.25$ & $37.22 \pm 2.53$ & 0.102 \\
Weight (kg) & $57.30 \pm 13.68$ & $59.12 \pm 13.09$ & 0.498 \\
Height (m) & $1.60 \pm 0.09$ & $1.61 \pm 0.09$ & 0.880 \\
Body mass index (kg/m²) & $22.23 \pm 4.77$ & $22.86 \pm 4.49$ & 0.499 \\
Serum melatonin at & $17.44 \pm 3.43$ & $17.10 \pm 3.71$ & 0.643 \\
14:00 hr (pg/ml) & & & \\
Serum melatonin at & $53.23 \pm 19.45$ & $54.66 \pm 18.77$ & 0.710 \\
2:00 hr (pg/ml) & & & \\
Serum AANAT (ng/ml) & $20.47 \pm 6.30$ & $18.33 \pm 7.35$ & 0.121 \\
Urinary melatonin & $0.91 \pm 0.33$ & $0.80 \pm 0.29$ & 0.102 \\
(pg/ml) & & & \\
Serum GAP-43 (ng/ml) & $2.94 \pm 0.76$ & $3.17 \pm 0.91$ & 0.185 \\
Total PANSS & $95.22 \pm 27.12$ & $87.34 \pm 23.28$ & 0.122 \\
PSQl & $10.40 \pm 2.96$ & $10.14 \pm 3.04$ & 0.666 \\
\hline
\end{tabular}

Values are presented as mean \pm standard deviation.

AANAT, arylalkylamine N-acetyltransferase; GAP-43, growth-associated protein 43; PANSS, Positive and Negative Syndrome Scale; PSQI, Pittsburgh sleep quality index. Unpaired $t$ test/Fisher's exact test. baseline suggesting homogeneity of the study groups (Table 1). The overall mean age of the participants was 35 years and $43 \%$ were female. As the number of subjects completing the study $(n=81)$ was less than the estimated sample size $(n=84)$, we have done post-hoc power analysis and found that the power achieved is more than $80 \%$.

\section{Change in Serum Melatonin at 2:00 Hours}

In haloperidol group, serum melatonin decreased significantly from $53.03 \mathrm{pg} / \mathrm{ml}$ to $50.61 \mathrm{pg} / \mathrm{ml}$ ( $p=0.008$ ) and similarly, in the risperidone group, there was a significant decrease from 56.27 to $52.86 \mathrm{pg} / \mathrm{ml}(p=0.021)$. The mean changes in both the groups were compared by unpaired $t$ test and found to be not significant $(p=0.558)$ (Table 2).

\section{Change in Serum Melatonin at 14:00 Hours}

In haloperidol group, serum melatonin decreased from $17.38 \mathrm{pg} / \mathrm{ml}$ to $17.11 \mathrm{pg} / \mathrm{ml}(p=0.479)$ and similarly, in the risperidone group, there was a decrease from 16.84 to $16.36 \mathrm{pg} / \mathrm{ml}(p=0.328)$. The mean changes in both the groups were compared by unpaired $t$ test and found to be not significant ( $p=0.745$ ) (Table 2).

\section{Change in Urinary Melatonin}

In haloperidol group, urinary melatonin decreased significantly from $0.90 \mathrm{pg} / \mathrm{ml}$ to $0.85 \mathrm{pg} / \mathrm{ml}(p=0.005)$ and similarly in risperidone group, there was a significant decrease from 0.80 to $0.75 \mathrm{pg} / \mathrm{ml}(p=0.014)$. The mean changes in both the groups were compared by unpaired $t$ test and found to be not significant $(p=0.934)$ (Table 2).

\section{Change in Serum AANAT}

In haloperidol group, serum AANAT decreased from $20.61 \mathrm{ng} / \mathrm{ml}$ to $20.07 \mathrm{ng} / \mathrm{ml}(p=0.344)$ and similarly, in the risperidone group, there was a decrease from 19.05 to $18.36 \mathrm{ng} / \mathrm{ml}(p=0.367)$. The mean changes in both the groups were compared by unpaired $t$ test and found to be not significant ( $p=0.876$ ) (Table 2).

\section{Change in Serum GAP-43}

In haloperidol group, serum GAP-43 decreased significantly from $2.92 \mathrm{ng} / \mathrm{ml}$ to $2.72 \mathrm{ng} / \mathrm{ml}(p<0.001)$ and similarly in the risperidone group, there was a significant decrease from 3.19 to $3.00 \mathrm{ng} / \mathrm{ml}(p<0.001)$. The mean changes in both the groups were compared by unpaired 


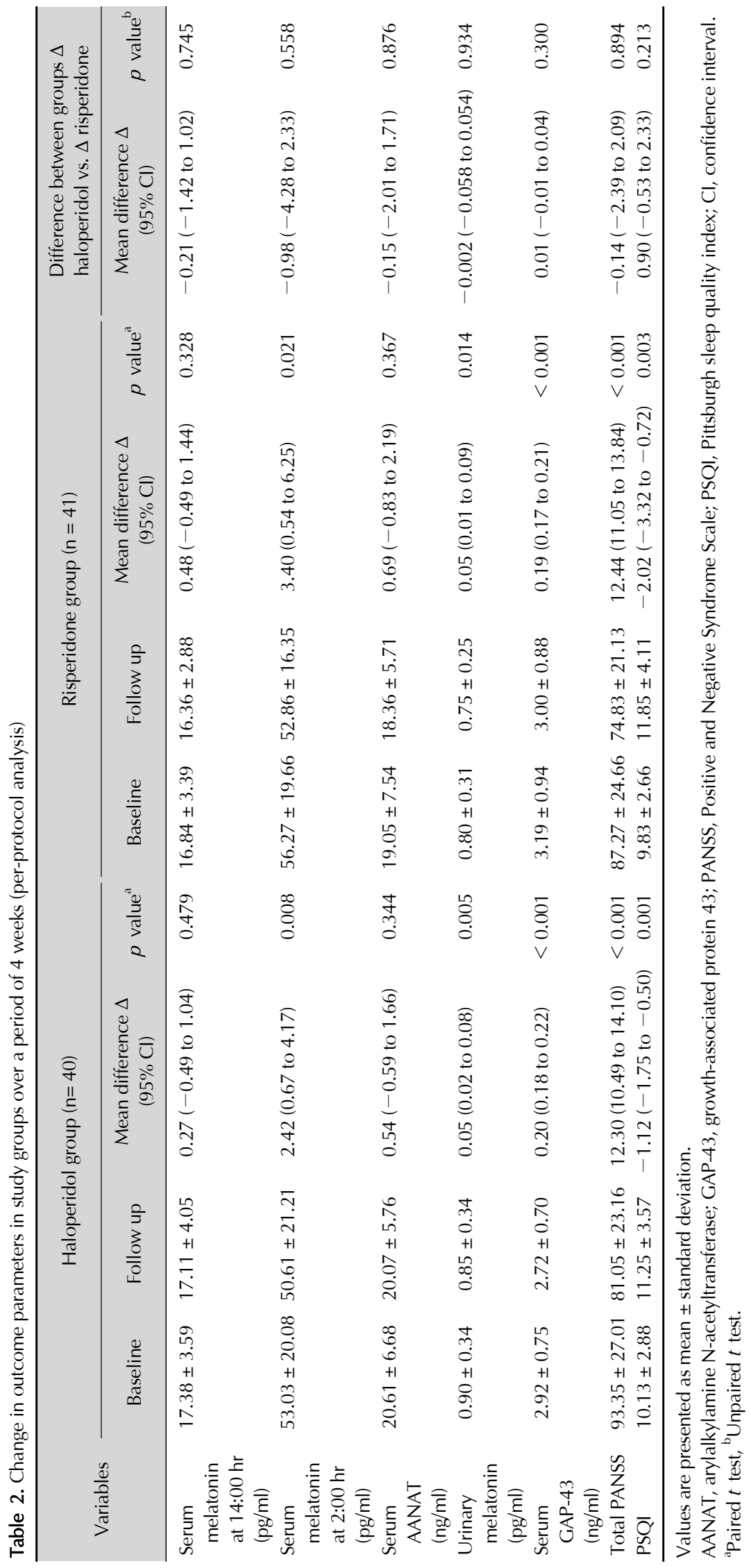




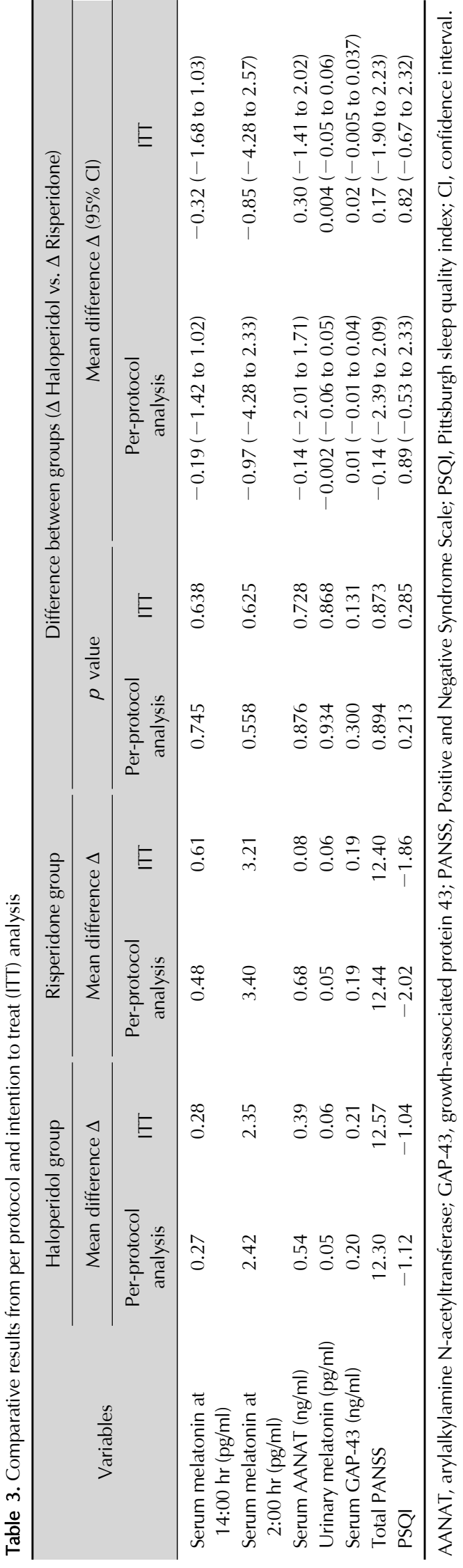

$t$ test and found to be not significant $(p=0.30)$ (Table 2).

\section{Change in PSQI Score}

In the haloperidol group, PSQI score increased significantly from 10.13 to $11.25(p=0.001)$ and similarly in the risperidone group, there was a significant increase from 9.83 to $11.85(p=0.003)$. The mean changes in both the groups were compared by unpaired $t$ test and found to be not significant ( $p=0.213$ ) (Table 2). PSQI score deteriorated in 29 patients $(73 \%)$ in the haloperidol group and 33 patients $(80 \%)$ in the risperidone group.

\section{Change in PANSS Score}

PANSS score in all three domains decreased significantly in both the study groups. In the haloperidol group, total PANSS score decreased significantly from 93.35 to $81.05(p<0.001)$ and similarly in the risperidone group, there was a significant decrease from 87.27 to 74.83 ( $p<$ $0.001)$. The mean changes in both the groups were compared by unpaired $t$ test and found to be not significant $(p=$ 0.894) (Table 2).

\section{Intention to Treat Analysis}

For ITT analysis, the missing values were replaced using multiple imputation techniques. ITT analysis was done for all outcome measures. The results were found to be similar to the per-protocol analysis (Table 3).

\section{Correlation Analysis}

Serum melatonin at 2:00 hours and PSQI score at baseline were found to be inversely correlated. Pearson's correlation between Serum melatonin at 2:00 hours and PSQI score was significant $(r=-0.48 ; 95 \%$ confidence interval: -0.615 to $-0.308 ; p<0.001$ ) (Fig. 2).

\section{Regression Analysis}

A linear regression analysis was done to assess four independent variables like age, body mass index, the season of enrolment of patients and smoking status as a predictor of serum melatonin at 2:00 hours. The equation calculated was not found to be significant. $F(4,96)=$ $0.674, p=0.611$, with an $\mathrm{R}^{2}=0.028$. The standardized beta coefficient and $p$ value for the independent variables are mentioned in Table 4. 


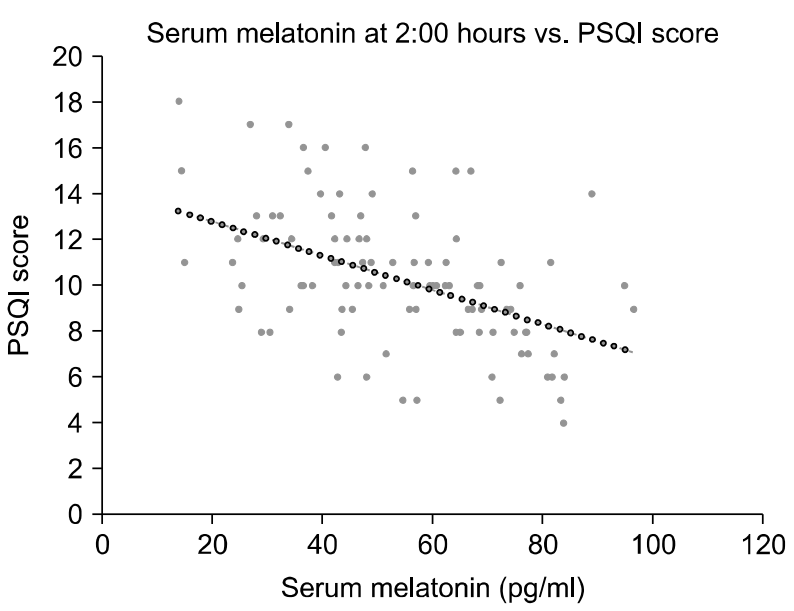

Fig. 2. Correlation between serum melatonin at 2:00 hours and PSQI score at baseline.

PSQI, Pittsburgh sleep quality index.

\section{Safety Evaluation}

The adverse events were determined by non-directive questioning to the patients and relatives and the severity of the adverse drug reaction (ADR) was evaluated according to the WHO toxicity grading scale. The study drugs were discontinued only if the patients developed grade 3 or 4 ADR. In the haloperidol group, 6 patients were excluded from the study as they developed extrapyramidal symptoms. Anticholinergic effects were complained by 5 patients in the haloperidol group whereas 4 patients from the risperidone group complained of dizziness. As these adverse effects were grade 1 or 2, study drugs were continued.

\section{DISCUSSION}

New drug targets for the treatment of schizophrenia are coming up but even today the first-line treatment is based on the monoamine hypothesis. The conventional antipsychotic drugs are effective in controlling the cardinal symptoms of schizophrenia but have several limitations [37]. As sleep and circadian disruptions play a critical role in mortality and morbidity $[9,38]$, this study was conducted to evaluate the adequacy of conventional antipsychotics in improving sleep and circadian rhythm disturbances.

In both the study groups, serum melatonin level at 2:00 hours was lower than the normal physiological level of melatonin [39]. Our study result is supported by the previous studies by Fanget et al. [17] and Monteleone et al.
Table 4. Standardized beta coefficient and $p$ value for independent variables

\begin{tabular}{lcrc}
\hline \multicolumn{1}{c}{$\begin{array}{c}\text { Independent } \\
\text { variable }\end{array}$} & $\begin{array}{c}\text { Standardized } \\
\text { beta coefficient }\end{array}$ & $t$ & $p$ value \\
\hline Age & -0.056 & -0.538 & 0.59 \\
Body mass index & 0.120 & 1.162 & 0.25 \\
Smoking status & 0.033 & 0.315 & 0.75 \\
Season of enrolment & -0.111 & -1.081 & 0.28 \\
\hline
\end{tabular}

[19]. Even after 4 weeks of therapy with conventional antipsychotic drugs (haloperidol, risperidone), there was a statistically significant decrease in serum melatonin and urinary melatonin in both groups. In a previous study by Robinson et al. [18] an obliterated nocturnal melatonin rise was evidenced, and the trend remained unchanged despite two months of drug treatment with neuroleptics. Another study by Mann et al. [33] could not find any significant change in serum melatonin after treatment with olanzapine for 4 weeks. Monteleone et al. [19] did not find any significant difference in the secretory pattern of melatonin between drug-free and drug-treated patients. So, the result of our study corroborates with previous studies and it is evident that antipsychotic drug treatment cannot improve the status of serum melatonin, rather there can be deterioration as found in the present study. AANAT, the penultimate enzyme in melatonin synthesis, controls rhythm in melatonin production and essential for the function of the circadian clock that influences sleep. In our study groups, we found a marginal decrease in serum AANAT. Though statistically non-significant, this finding indicates that antipsychotic drugs cannot improve the level of AANAT. GAP-43 being a protein involved in neurite formation, regeneration and synaptic plasticity, increase in GAP-43 level may be considered as an improvement in the pathobiology of the disease. In our study, in both the groups there was a significant decrease in GAP-43 from baseline even after treatment with antipsychotics. In a previous study by Eastwood et al. [40] suggested that haloperidol does not produce a sustained alternation of neural plasticity. Our study result also indicates that antipsychotic therapy could not improve GAP-43 level and in turn synaptic and neuronal plasticity. The decrease in serum GAP-43 may be a due decrease in melatonin secretion as melatonin has been found to upregulate GAP-43 in the study done by Juan et al. [31]. PSQI score was found to increase significantly in both groups 
suggesting deterioration of the quality of sleep even under treatment with antipsychotics. In a previous study by Yamashita et al. [41], the atypical antipsychotic drugs were found to be beneficial to the quality of sleep in elderly patients with schizophrenia. The findings of our study are different probably because of the younger population in our study (mean age of 35 in the present study vs. 61 in the study by Yamashita). Symptom severity estimated through PANSS scoring was found to be improved in both the groups. In our study, we found that even after treatment with antipsychotic drugs, the sleep and circadian rhythm disturbances deteriorated. Hence, it is evident that haloperidol and risperidone have no significant favourable effect on biosynthesis of melatonin and GAP-43. The worsening may be due to disease progression or due to another psychopathology or unknown mechanism of the antipsychotic drugs.

Melatonin exerts its physiological actions by interacting with MT1 and MT2 receptors in the suprachiasmatic nucleus (SCN) which in turn projects to reticular thalamic nuclei. MT1 receptor activation inhibits neuronal firing in SCN and MT2 receptor phase shifts the circadian rhythm. Role of benzodiazepines have been investigated but they were found to depress nocturnal melatonin secretion [42-45]. Z-compounds also at higher doses showed a negative linear correlation between the doses of z-compound and melatonin concentration, suggesting the possibility of suppression at higher doses or with chronic administration [46]. So, in this clinical scenario, add-on melatonin or add-on melatonin agonist may be helpful in ameliorating sleep and circadian disturbances [47].

\section{Limitations}

Serum melatonin has been estimated at two-time points but estimation at repeated intervals could have clearly delineated and detect the phase shift effect and phase response curve of the disease and the therapy. As the sampling was done after hospitalizing patients for one day, environmental and light conditions of the ward may have affected the circadian rhythm to a certain extent. Sampling after an adaptation to a new environment could have been done. Even adrenergic level from intravenous puncture and posture also can influence the level of melatonin. The present study has not investigated into the mechanistic profile of the antipsychotics or psychopathology of the disease and hence, could not answer the reason of de- terioration of sleep and circadian rhythm disturbance in schizophrenia.

In conclusion, monotherapy with haloperidol and risperidone can achieve symptomatic improvement but cannot improve sleep and circadian rhythm disturbances in schizophrenia. In this clinical scenario, early intervention with add-on melatonin or add-on melatonin agonist may be investigated in future studies.

\section{Acknowledgments}

This research was supported by Institutional Research Grant (IRG) from All India Institute of Medical Sciences, Bhubaneswar, India.

The authors would like to express heartfelt thanks to Dr. Debasish Hota, D.M., and Dr. Anand Srinivasan, D.M., for their valuable suggestions, active help, and encouragement.

\section{- Conflicts of Interest}

No potential conflict of interest relevant to this article was reported.

\section{Author Contributions}

The conception or design of the research: Rituparna Maiti, Biswa Ranjan Mishra. The analysis, or interpretation of data: Rituparna Maiti, Monalisa Jena, Archana Mishra, Santanu Nath. Drafting the manuscript: Rituparna Maiti, Monalisa Jena, Archana Mishra. Final approval of the version to be published: Rituparna Maiti, Biswa Ranjan Mishra, Monalisa Jena, Archana Mishra, Santanu Nath. Revising the manuscript critically for important intellectual content: Biswa Ranjan Mishra, Santanu Nath. Accountable for all aspects of the work in ensuring that questions related to the accuracy or integrity of any part of the work are appropriately investigated and resolved.

\section{ORCID}

Rituparna Maiti https://orcid.org/0000-0003-4063-9178 Biswa Ranjan Mishra

https://orcid.org/0000-0003-0537-9454

Monalisa Jena https://orcid.org/0000-0001-6244-2988

Archana Mishra https://orcid.org/0000-0001-8837-299X

Santanu Nath https://orcid.org/0000-0001-5357-3783

\section{REFERENCES}

1. Lieberman JA, Koreen AR. Neurochemistry and neuroendo- 
crinology of schizophrenia: a selective review. Schizophr Bull 1993;19:371-429.

2. Tandon R, Keshavan MS, Nasrallah HA. Schizophrenia, "just the facts": what we know in 2008 part 1: overview. Schizophr Res 2008;100:4-19.

3. Lieberman JA, Girgis RR, Brucato G, Moore H, Provenzano F, Kegeles L, et al. Hippocampal dysfunction in the pathophysiology of schizophrenia: a selective review and hypothesis for early detection and intervention. Mol Psychiatry 2018;23: 1764-1772.

4. Yates NJ. Schizophrenia: the role of sleep and circadian rhythms in regulating dopamine and psychosis. Rev Neurosci 2016;27:669-687.

5. Tam SK, Pritchett D, Brown LA, Foster RG, Bannerman DM, Peirson SN. Sleep and circadian rhythm disruption and recognition memory in schizophrenia. Methods Enzymol 2015; 552:325-349.

6. Cosgrave J, Wulff K, Gehrman P. Sleep, circadian rhythms, and schizophrenia: where we are and where we need to go. Curr Opin Psychiatry 2018;31:176-182.

7. Benson KL. Sleep in schizophrenia: pathology and treatment. Sleep Med Clin 2015; 10:49-55.

8. Kaskie RE, Graziano B, Ferrarelli F. Schizophrenia and sleep disorders: links, risks, and management challenges. Nat Sci Sleep 2017;9:227-239.

9. Kamath J, Virdi S, Winokur A. Sleep disturbances in schizophrenia. Psychiatr Clin North Am 2015;38:777-792.

10. Klingaman EA, Palmer-Bacon J, Bennett ME, Rowland LM. Sleep disorders among people with schizophrenia: emerging research. Curr Psychiatry Rep 2015;17:79.

11. Robillard R, Rogers NL, Whitwell BG, Lambert T. Are cardiometabolic and endocrine abnormalities linked to sleep difficulties in schizophrenia? A hypothesis driven review. Clin Psychopharmacol Neurosci 2012;10:1-12.

12. Bromundt V, Köster M, Georgiev-Kill A, Opwis K, Wirz-Justice A, Stoppe G, et al. Sleep-wake cycles and cognitive functioning in schizophrenia. Br J Psychiatry 2011;198:269-276.

13. Wulff K, Dijk DJ, Middleton B, Foster RG, Joyce EM. Sleep and circadian rhythm disruption in schizophrenia. BrJ Psychiatry 2012;200:308-316.

14. Morera-Fumero AL, Abreu-Gonzalez P. Role of melatonin in schizophrenia. Int J Mol Sci 2013;14:9037-9050.

15. Anderson G, Maes M. Melatonin: an overlooked factor in schizophrenia and in the inhibition of anti-psychotic side effects. Metab Brain Dis 2012;27:113-119.

16. Ferrier IN, Arendt J, Johnstone EC, Crow TJ. Reduced nocturnal melatonin secretion in chronic schizophrenia: relationship to body weight. Clin Endocrinol (Oxf) 1982;17:181-187.

17. Fanget F, Claustrat B, Dalery J, Brun J, Terra JL, Marie-Cardine $\mathrm{M}$, et al. Nocturnal plasma melatonin levels in schizophrenic patients. Biol Psychiatry 1989;25:499-501.

18. Robinson S, Rosca P, Durst R, Shai U, Ghinea C, Schmidt U, et al. Serum melatonin levels in schizophrenic and schizo- affective hospitalized patients. Acta Psychiatr Scand 1991; 84:221-224.

19. Monteleone P, Natale M, La Rocca A, Maj M. Decreased nocturnal secretion of melatonin in drug-free schizophrenics: no change after subchronic treatment with antipsychotics. Neuropsychobiology 1997;36:159-163.

20. Sandyk R, Kay SR. Pineal melatonin in schizophrenia: a review and hypothesis. Schizophr Bull 1990;16:653-662.

21. Owen F, Ferrier IN, Poulter M, Crow TJ. Hydroxy-indole-omethyltransferase activity in human pineals: a comparison of controls and schizophrenics. Clin Endocrinol (Oxf) 1983;19: 313-317.

22. Chambers JS, Thomas D, Saland L, Neve RL, Perrone-Bizzozero NI. Growth-associated protein 43 (GAP-43) and synaptophysin alterations in the dentate gyrus of patients with schizophrenia. Prog Neuropsychopharmacol Biol Psychiatry 2005;29:283290.

23. Benowitz LI, Routtenberg A. GAP-43: an intrinsic determinant of neuronal development and plasticity. Trends Neurosci 1997;20:84-91.

24. Perrone-Bizzozero NI, Sower AC, Bird ED, Benowitz LI, Ivins $\mathrm{KJ}$, Neve RL. Levels of the growth-associated protein GAP-43 are selectively increased in association cortices in schizophrenia. Proc Natl Acad Sci U S A 1996;93:14182-14187.

25. Blennow K, Bogdanovic N, Gottfries CG, Davidsson P. The growth-associated protein GAP-43 is increased in the hippocampus and in the gyrus cinguli in schizophrenia. I Mol Neurosci 1999;13:101-109.

26. Sower AC, Bird ED, Perrone-Bizzozero NI. Increased levels of GAP-43 protein in schizophrenic brain tissues demonstrated by a novel immunodetection method. Mol Chem Neuropathol 1995;24:1-11.

27. Weickert CS, Webster MJ, Hyde TM, Herman MM, Bachus SE, Bali G, et al. Reduced GAP-43 mRNA in dorsolateral prefrontal cortex of patients with schizophrenia. Cereb Cortex 2001;11:136-147.

28. Webster MJ, Shannon Weickert C, Herman MM, Hyde TM, Kleinman JE. Synaptophysin and GAP-43 mRNA levels in the hippocampus of subjects with schizophrenia. Schizophr Res 2001;49:89-98.

29. Hakak Y, Walker JR, Li C, Wong WH, Davis KL, Buxbaum JD, et al. Genome-wide expression analysis reveals dysregulation of myelination-related genes in chronic schizophrenia. Proc Natl Acad Sci U S A 2001;98:4746-4751.

30. Eastwood SL, Harrison PJ. Hippocampal and cortical growthassociated protein-43 messenger RNA in schizophrenia. Neuroscience 1998;86:437-448.

31. Juan WS, Huang SY, Chang CC, Hung YC, Lin YW, Chen TY, et al. Melatonin improves neuroplasticity by upregulating the growth-associated protein-43 (GAP-43) and NMDAR postsynaptic density-95 (PSD-95) proteins in cultured neurons exposed to g/utamate excitotoxicity and in rats subjected to transient focal cerebral ischemia even during a long-term recov- 
ery period. J Pineal Res 2014;56:213-223.

32. Shen YC, Tsai HM, Cheng MC, Hsu SH, Chen SF, Chen $\mathrm{CH}$. Genetic and functional analysis of the gene encoding GAP-43 in schizophrenia. Schizophr Res 2012;134:239-245.

33. Mann K, Rossbach W, Müller MJ, Müller-Siecheneder F, Pott $\mathrm{T}$, Linde I, et al. Nocturnal hormone profiles in patients with schizophrenia treated with olanzapine. Psychoneuroendocrinology 2006;31:256-264.

34. Kay SR, Fiszbein A, Opler LA. The positive and negative syndrome scale (PANSS) for schizophrenia. Schizophr Bull 1987; 13:261-276.

35. Buysse DJ, Reynolds CF 3rd, Monk TH, Berman SR, Kupfer DJ. The Pittsburgh Sleep Quality Index: a new instrument for psychiatric practice and research. Psychiatry Res 1989;28:193213.

36. Mills MH, King MG, Keats NG, McDonald RA. Melatonin determination in human urine by high-performance liquid chromatography with fluorescence detection. J Chromatogr 1986; 377:350-355.

37. Stępnicki P, Kondej M, Kaczor AA. Current concepts and treatments of schizophrenia. Molecules 2018;23:2087.

38. Monti JM, BaHammam AS, Pandi-Perumal SR, Bromundt V, Spence DW, Cardinali DP, et al. Sleep and circadian rhythm dysregulation in schizophrenia. Prog Neuropsychopharmacol Biol Psychiatry 2013;43:209-216.

39. Zhdanova IV, Wurtman RJ, Balcioglu A, Kartashov AI, Lynch $\mathrm{HJ}$. Endogenous melatonin levels and the fate of exogenous melatonin: age effects. J Gerontol A Biol Sci Med Sci 1998;53: B293-B298.

40. Eastwood SL, Heffernan J, Harrison PJ. Chronic haloperidol treatment differentially affects the expression of synaptic and neuronal plasticity-associated genes. Mol Psychiatry 1997;2: 322-329.

41. Yamashita H, Mori K, Nagao M, Okamoto Y, Morinobu S, Yamawaki S. Influence of aging on the improvement of subjective sleep quality by atypical antipsychotic drugs in patients with schizophrenia: comparison of middle-aged and older adults. Am J Geriatr Psychiatry 2005;13:377-384.

42. Hajak G, Rodenbeck A, Bandelow B, Friedrichs S, Huether G, Rüther E. Nocturnal plasma melatonin levels after flunitrazepam administration in healthy subjects. Eur Neuropsychopharmacol 1996;6:149-153.

43. Kabuto M, Namura I, Saitoh Y. Nocturnal enhancement of plasma melatonin could be suppressed by benzodiazepines in humans. Endocrinol Jpn 1986;33:405-414.

44. Monteleone P, Forziati D, Orazzo C, Maj M. Preliminary observations on the suppression of nocturnal plasma melatonin levels by short-term administration of diazepam in humans. J Pineal Res 1989;6:253-258.

45. McIntyre IM, Burrows GD, Norman TR. Suppression of plasma melatonin by a single dose of the benzodiazepine alprazolam in humans. Biol Psychiatry 1988;24:108-112.

46. Norman TR, Piccolo J, Voudouris N, Burrows GD. The effect of single oral doses of zopiclone on nocturnal melatonin secretion in healthy male volunteers. Prog Neuropsychopharmacol Biol Psychiatry 2001;25:825-833.

47. Mishra A, Maiti R, Mishra BR, Jena M, Nath S, Sahu P. Effect of add-on ramelteon therapy on sleep and circadian rhythm disruption in patients with schizophrenia: a randomized controlled trial. Eur Neuropsychopharmacol 2020;31:109-118. 